\title{
Two active $X$ Chromosomes and methylation of the imprinted gene in human parthenogenetic embryonic stem cell line
}

Xiaofang Sun ${ }^{1}$, Shaorong Gao ${ }^{2}$, Xiaolin Long ${ }^{1}$, Yifei Yin ${ }^{1}$, Yonghua Jiang ${ }^{1}$, Wenhong Zhang ${ }^{1}$, Yuhong Zheng, Hongzi Du ${ }^{1}$, Huangsheng Chang, Weiqiang Liu, Weihua Wang ${ }^{1}$, Guohong Xiao ${ }^{1}$

${ }^{1}$ Institute of Gynecology and Obstetrics, The Third Affiliated Hospital of Guangzhou Medical College, Duobao Road, Guangzhou, China ${ }^{2}$ National Institute of Biological Sciences, Beijing China

Parthenogenetic embryonic stem cells (PESC) play important roles in the study of gene function and may also be important in the cell therapy.In the present study, one pluripotent human embryonic stem cell (hPESC) line was isolated from parthenogenetic blastocysts. These cells remained undifferentiated state after more than 38 passages and still maintained a normal 46, XX karyotype. The hPESC display a similar morphology as compared with traditional human embryonic stem cells (hESC) derived from fertilized eggs, such as forming colonies with tightly packed cells, prominent nucleoli, and a small cytoplasm to nucleus ratio. The hPESC line expressed traditional hESC markers, such as SSEA-3, SSEA-4, TRA-1-60, TRA-1-81, and did not express SSEA-1. These cells also showed positive expression of transcription factor OCT-4, Nanog, Sox2 and a high level of alkaline phosphatase activity. DNA profiling of hPESC line indicated that it is MHC matched with the oocyte donor. In order to identify the methylation status in undifferentiated hPESC line, DNA methylation pattern of the imprint control (IC) region of the SNRPN-gene was examined, and only methylated maternal SNRPN -gene was detected in this study. Examination of $X$ chromosome inactivation status showed that both $X$ chromosomes are active, indicating that DNA methylation on both $\mathrm{X}$ chromosomes is globally reduced in hPESC line.Furthermore, the monoallelic expression of parentally expressed imprinted gene IGF2 and maternally expressed imprinted gene H19 were also analyzed, the results provided the further evidence of the parthenogenetic origin of the hPESC line. In conclusion, we have generated a phESC line and this cell line can be differentiated into various types of cells, which may be of great value in the future application on the treatment of human diseases and for further study of epigenetic mechanisms.

Keywords: human parthenogenetic stem cell line (hPESC), imprinted gene, DNA methylation, X chromosome, active, epigenetic mechanisms

Cell Research (2008) 18:s35. doi: 10.1038/cr.2008.125; published online 4 August 2008

Correspondence: Xiaofang Sun

E-mail:xiaofangsun@hotmail.com 